Artículo

\title{
Caracterización apícola en la región sierra centro-norte de Veracruz: contexto y trashumancia
}

\author{
Giovanni Luna Chontal ${ }^{1}$ \\ Juan Gabriel Roque Peña ${ }^{1}$ \\ Eduardo Fernández Echeverría ${ }^{2}$ \\ Eduardo Martínez Mendoza ${ }^{3}$ \\ Ulises Asrrael Díaz Zorrilla ${ }^{4}$ \\ Gregorio Fernández Lambert ${ }^{1 \S}$
}

${ }^{1}$ Instituto Tecnológico Superior de Misantla. Carretera a Loma del Cojolite km 1.8, Misantla, Veracruz, México. CP. 93821. (glunac@itsm.edu.mx; jgroquep@itsm.edu.mx). ${ }^{2}$ Instituto Tecnológico Superior de Zacapoaxtla. Carretera a Acuaco-Zacapoaxtla km 8, Totoltepec, Zacapoaxtla, Puebla (iiefernandez@ hotmail.com). CP. 73680. ${ }^{3}$ Universidad del Istmo-Campus Tehuantepec. Ciudad Universitaria S/N, Barrio Santa Cruz, $4^{\text {ta }}$ Sección Santo Domingo Tehuantepec, Oaxaca, México. CP. 70760. (ed. mtzm@hotmail.com). ${ }^{4}$ Campo Experimental Ixtacuaco-INIFAP. Carretera Federal Martínez de la TorreTlapacoyan km 4.5, Veracruz, México. AP. 162. (diaz.ulises@inifap.gob.mx).

${ }^{\S}$ Autor para correspondencia: gfernandezl@itsm.edu.mx.

\section{Resumen}

Este artículo presenta el resultado de una investigación realizada de agosto 2015 a noviembre 2016, para caracterizar la producción apícola y actividad trashumante de los municipios de Misantla, Yecuatla, Colipa, Juchique de Ferrer y Tenochtitlan. Se realizaron entrevistas con especialistas, expertos y productores de miel. Se encontró que la actividad apícola la realizan al menos 500 familias con experiencia promedio de 22 años, con sistemas trashumantes semi extensivos y producción promedio de $24 \mathrm{~kg}$ de miel por colmena, de los cuales $9 \%$ tienen más de 500 colmenas bajo esquemas semi-tecnificados. Entre estos sistemas el 10\% de apicultores han invertido en la diferenciación de los productos de la colmena. El volumen de miel generado con sistemas de producción de una abeja reina por colmena es al menos $90 \mathrm{t}_{\text {año }}{ }^{-1}$, con valor económico de \$ 174 000.00 (US\$). Se ha encontrado que $90 \%$ de los apicultores de la región trashuman entre lugares de dicha región y $23 \%$ de ellos lo realizan a otra entidad. Por otra parte, también el acceso y permiso a terrenos son factores que definen las rutas trashumantes e identificación de zonas aptas apiarios. Esta investigación aporta un mapa de zonas utilizadas como asentamientos de apiarios, como trabajo de investigación, sus resultados merecen la atención futuras investigaciones para identificar zonas espaciales mediante sistemas de geoposicionamiento, así como un programa de trashumancia para dichos emplazamientos en función de calendarios de floración.

Palabras clave: abejas, apiarios, colmena, miel de abeja.

Recibido: marzo de 2019

Aceptado: junio de 2019 


\section{Introducción}

La región serrana centro-norte del Estado de Veracruz posee una diversidad territorial en ecosistemas, y amplia actividad agrícola en el cultivo de café, forrajes, cítricos, entre otros cultivos de menor escala los cuales ofrecen altas posibilidades para el ejercicio de la actividad apícola. La actividad apícola está inmersa con las estaciones de floración caracterizadas por las variaciones del clima y flora de cada región la cual permite que la apicultura se convierta en la tercera fuente generadora de divisas en el sector agropecuario de México; sin embargo, dicho potencial biótico, contrasta con el bajo nivel de producción de miel de abeja comparado en el orden internacional (Magaña y Leyva, 2011).

En este sentido, toman importancia las prácticas apícolas dentro de las que sobresale la trashumancia, como una estrategia de producción en dicho sector. La producción de miel en la región serrana centro-norte del estado de Veracruz ha generado una actividad de alto valor económico en la última década como se muestra en la Figura 1 con una producción en el año 2016 de $82 \mathrm{t}$, la cual puede favorecerse en rendimiento de producción con el uso de información que apoye en las decisiones de asentamientos de colmenas en mejores zonas de pecoreo.

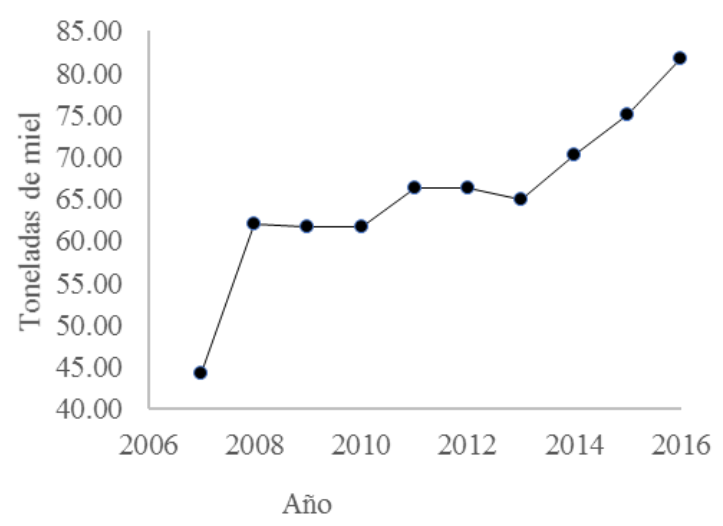

Figura 1. Producción en toneladas de miel en la región de Misantla, Veracruz. Fuente: SAGARPA (2010).

La actividad de trashumancia se encuentra estrechamente ligada al ambiente y recursos naturales de la zona que el productor utiliza para instalar sus apiarios, de manera que el conocimiento de la vegetación espacial y temporal es importante para planificar el manejo y movilidad de las colmenas. Sin embargo, debe reconocerse las zonas de floración han sido modificadas en la última década por diferentes factores, entre ellos, la tala clandestina o el incremento de extensión de cultivos intensivos, que modifican los sistemas bióticos con impacto en bajos rendimientos de producción de miel por colmena (Bellarby et al., 2008; SAGARPA, 2010).

No obstante que la actividad apícola en México no es diferente a la que se realiza en otras partes del mundo, la tarea de identificar previamente áreas con aptitud apícola no es una actividad común en México, sino más bien es realizada por conocimiento familiar-hereditario. La trashumancia, en este sentido, se considera como una acción estratégica del apicultor para incrementar los rendimientos de producción de miel (Zoccali et al., 2017), en equilibrio con el recurso biótico del medio ambiente (Solomon, 1971; Roubik, 1995). 
La elección de la zona para el asentamiento de las colmenas considera, además de las técnicofinancieras la selectividad de la colmena para cierto tipo de flora de especies botánicas productoras de néctar y polen, aptas morfológicamente para ser explotadas por las abejas (González y Sánchez, 2008). Abou-Shaara et al. (2013) reportan que los riesgos de una inadecuada decisión de ubicación del apiario, resulta en menor rendimiento de producción de miel de la colmena; por lo tanto, la trashumancia implica invertir recursos de tiempo e infraestructura logística a la dinámica de esta actividad que busca un equilibrio ambiental para maximizar la producción de miel en función de la densidad de flora melífera y nectarífera y minimizar el recorrido de la abeja en su pecoreo; sin embargo, en los últimos años, cambios drásticos por efecto de variación del ambiente natural y por acciones de intervención humana, hace más complicada la ubicación de este tipo de lugares para el asentamiento de colmenas.

Esta investigación describe la actividad apícola y trashumante realizada en la región centro-norte de Veracruz, delimitada por Misantla (19 ${ }^{\circ} 55^{\prime}$ 51.86' latitud norte $96^{\circ} 51^{\prime}$ 6.09' longitud oeste); Yecuatla ( $19^{\circ} 51^{\prime}$ '57' ' latitud norte $96^{\circ} 46^{\prime} 36^{\prime}$ ' longitud oeste; Colipa (19 $55^{\circ}$ ' $25^{\prime}$ ' latitud norte $96^{\circ} 43^{\prime} 38^{\prime}$ ' longitud oeste; Juchique (19 $50^{\circ}$ ' $25^{\prime}$ ' latitud norte $96^{\circ} 41^{\prime} 41^{\prime}$ ' longitud oeste) y Tenochtitlan ( $19^{\circ} 48^{\prime} 27^{\prime}$ ' latitud norte $96^{\circ} 54^{\prime}$ 39' longitud oeste). La colindancia de estos municipios cubre $1159 \mathrm{~km}^{2}$, con una población aproximada de 101613 habitantes (INEGI, 2010).

\section{Materiales y métodos}

La investigación es de tipo transversal y descriptiva, soportada en fuentes primarias y secundarias con técnicos y expertos en el sector apícola, productores de miel, representantes y ex representantes de asociaciones de apicultores de la región de estudio. La información primaria se recoge apoyada de entrevistas no estructuradas personalizadas y un cuestionario diagnóstico, para recoger tanto los rendimientos de producción por temporada y cosecha, así como los elementos para trashumar.

Para reducir la ambigüedad de la información se utilizó el método Delphi en un panel de 7 expertos conformado por apicultores de la región de estudio con reconocida trayectoria como productor apícola. De una población registrada de 52 socios en la Asociación de Apicultores de Misantla (AAM), se entrevistaron 35 apicultores, mientras que aquellos no registrados en la AAM, se identificaron mediante la técnica de bola de nieve lineal durante el periodo del año 2015 y 2016.

Con esta técnica se amplió el rango de entrevista con apicultores de la región con baja incidencia o de difícil acceso, con el propósito de identificar el rasgo específico de la población de estudio para su categorización como apicultor. En ambos casos, las variables de estudio relacionadas con la cadena de producción de la miel: edad, actividad económica principal, experiencia en el ramo, número de colmenas por apiario, así como accesos a las zonas de asentamiento y su trashumancia.

Los datos de los cuestionarios fueron tratados mediante un procesamiento estadístico elemental para su representación cuantitativa. Las respuestas de entrevistas no estructuradas realizadas de forma personalizada se procesaron en frecuencia para reducirlas a categorías descriptivas y expresarlas porcentualmente. Este análisis de datos se realiza con la intención de interpretar los hallazgos importantes de la práctica apícola y trashumancia para caracterizar la apicultura en esta región de estudio. Con base a estos objetivos, se describieron mediante medidas de tendencia central y valores de proporción, según el caso, las variables de estudio de esta investigación. 
Para georreferenciar los sitios de asentamiento de apiarios se realizaron visitas in situ, apoyados con un dispositivo GPS modelo Garmín X15. Los puntos georreferenciados fueron procesados en el software Qgis Las Palmas, en su versión 2.18, software con licencia libre. La Figura 1 describe las zonas con aptitud apícola utilizadas actualmente por los apicultores de la región de estudio.

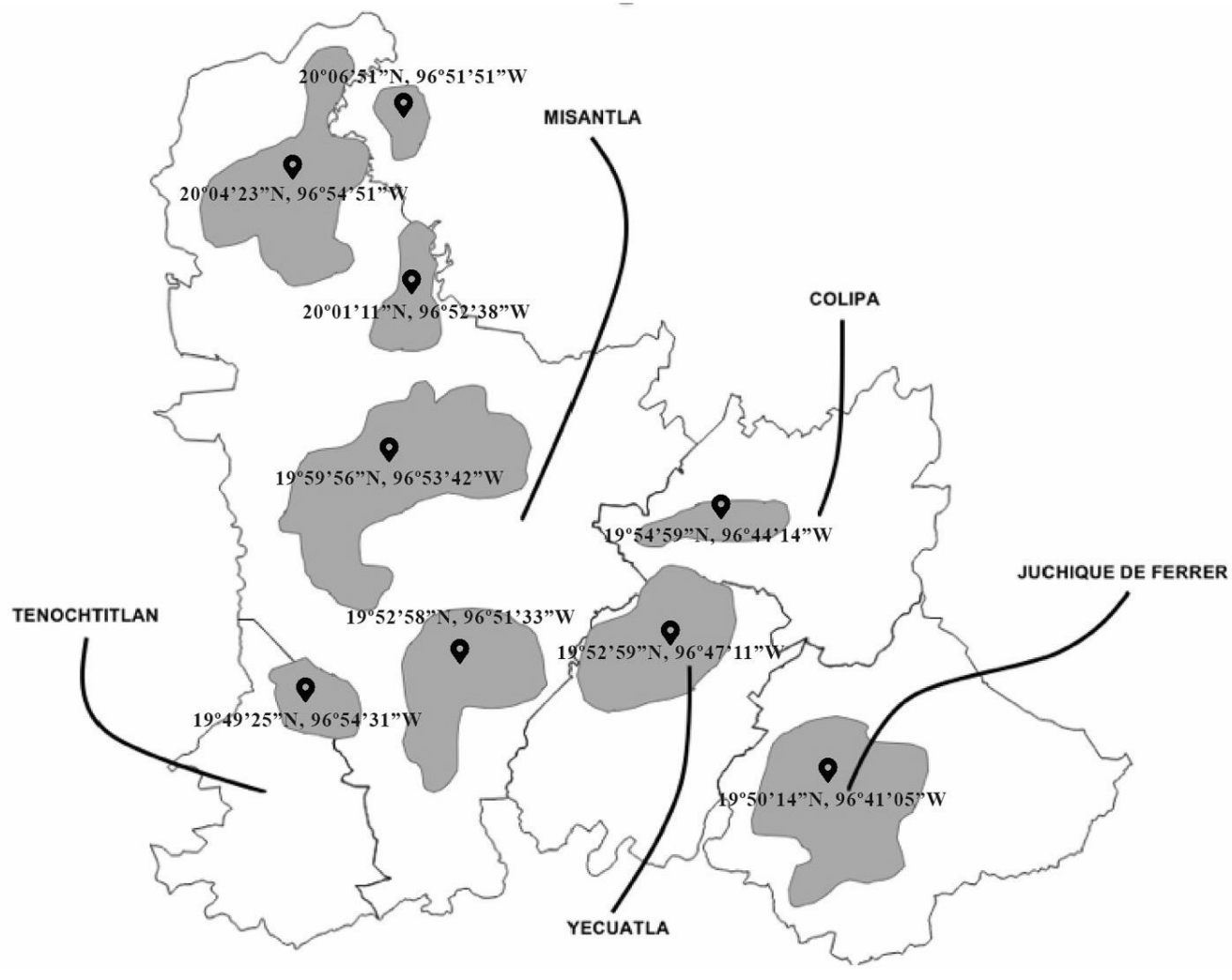

Figura 1. Zonas identificadas con actitud apícola por apicultores de la región intermunicipal de Misantla. Fuente: diseño propio a partir de la investigación de campo realizada de agosto 2015 a noviembre 2016.

\section{Resultados}

Los componentes naturales de la región de estudio generan un clima tropical cálido, cálidos subhúmedo, templado húmedo y de régimen térmico cálido con precipitaciones que varían en promedio de $2036 \mathrm{~mm}$ anuales. La temperatura media anual oscila en $22.7{ }^{\circ} \mathrm{C}$, con humedad relativa de $35 \%$ a $40 \%$. Los meses más fríos son diciembre y enero, el calor más intenso de abril a agosto. Las heladas se registran ocasionalmente en los meses de diciembre. Los fenómenos meteorológicos especiales como ciclones, tormentas eléctricas y nortes, que regularmente ocasionan fuertes lluvias, se registran en los meses de septiembre y octubre (INEGI, 2015).

La región de estudio se caracteriza por una topografía semi-regular en lomeríos y en pendientes mayores de 45 grados de inclinación, lo que permite la práctica de actividades agropecuarias con una barrera de árboles del trópico húmedo. El sistema productivo dominante es el ganadero y sus pastizales se mezclan con áreas dedicadas a la producción de cítricos (mandarina, naranja, limón, toronja y tangerina) que abarca grandes extensiones, principalmente en el sur del municipio de Misantla, con una gran presencia de plantaciones de café mayormente en sitios serranos. 
La zona intermunicipal cuenta con una hidrología enmarcada por la cuenca del río Nautla en la que se extiende el río Bobos, río Nautla, río Misantla, río Colipa y río Juchique que hacen de estas tierras aptas para las actividades agropecuarias (INEGI, 2015), mientras que las fuentes de agua de manantiales y arroyos, que descienden del cerro, crean un escenario propio para la práctica apícola.

El apicultor realiza actividades agrícolas y tiene a la apicultura como una segunda fuente de ingresos, ubicando sus colmenas geográficamente en su región tratando de abarcar la mayor floración posible. Normativamente en la Ley Apícola No. 830 del estado de Veracruz, un apiario debe conformarse con un mínimo de 20 colmenas, aún y cuando es común observar apiarios instalados con apenas dos colmenas o menos de 15.

Para lograr los mejores rendimientos de producción, los apicultores procuran colocar los apiarios a una distancia entre los $200 \mathrm{~m}$ respecto a los pasos peatonales, lugares públicos y casas habitación para guardar distancia a personas y animales domésticos; mientras que entre apiarios, los apicultores de la región estudio reportan colocarlos a $1 \mathrm{~km}$ de distancia para evitar el congestionamiento y la competencia entre las pecoreadoras por el suministro floral, lo que presume ocasionar el bajo rendimiento de producción de miel.

La Figura 2 muestra un estrato de 5 categorías: 1-50 colmenas, de 51-100 colmenas, de 101 a 300 colmenas, de 301 a 500 colmenas y más de 500 colmenas de un total de 120 apicultores entre asociados e independientes en la región de estudio. Esta categorización se realiza para estudios futuros de este sistema de producción, de los cuales, $85 \%$ de los apiarios se encuentran en terrenos ejidales y $15 \%$ en terrenos comunales.

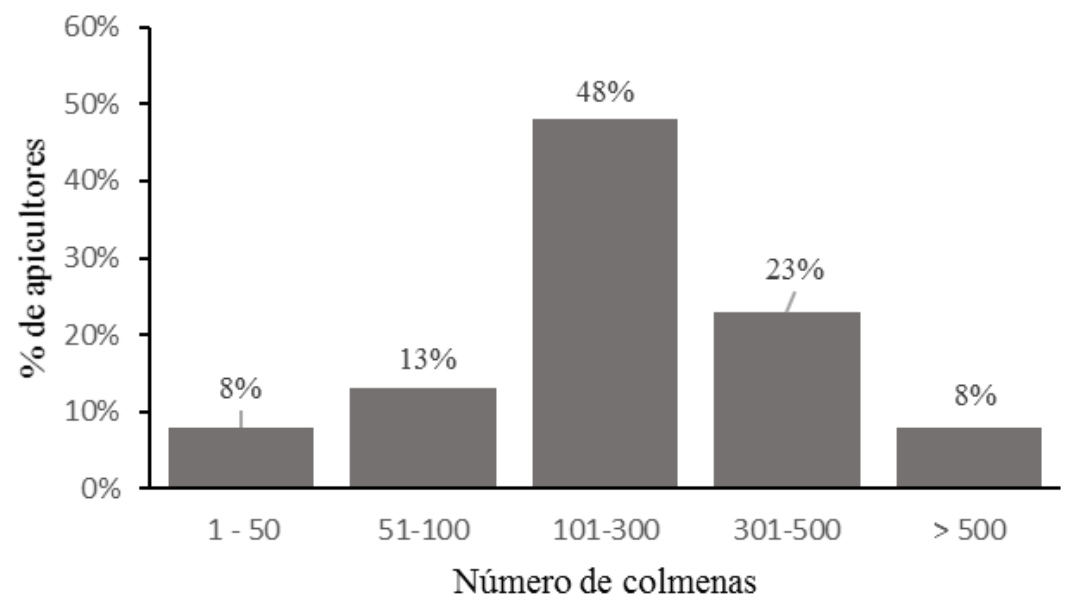

Figura 2. Estratificación de apicultores de la AAM acorde al número de colmenas. Fuente: diseño a partir de la investigación de campo realizada de agosto 2015 a noviembre 2016.

La Figura 3 describe que los caminos de acceso a los apiarios en la región de estudio, en lo general, $57 \%$ son rodadas, $29 \%$ carreteras asfaltadas en regulares condiciones de tránsito, $7 \%$ caminos y el resto son caminos reales.

Productores de la región de estudio, al igual que Visscher y Seeley (1982); Seeley (1995) y Beekman y Ratnieks (2001), reportan que en una colmena conviven y trabajan en colonia entre 10 000 y 120000 abejas que requieren de grandes insumos de néctar y polen de flores melíferas. Al 
respecto, la literatura reporta que las colonias pueden alimentarse de forma rutinaria cubriendo un área de entre 5-10 $\mathrm{km}^{2}$; sin embargo, en condiciones extremas se han reportado rangos mucho más grandes (Erickson et al., 1978). Este suceso es observado en lugares comerciales: paleterías, panaderías, cafeterías, en cada ciudad de los municipios objeto de estudio.

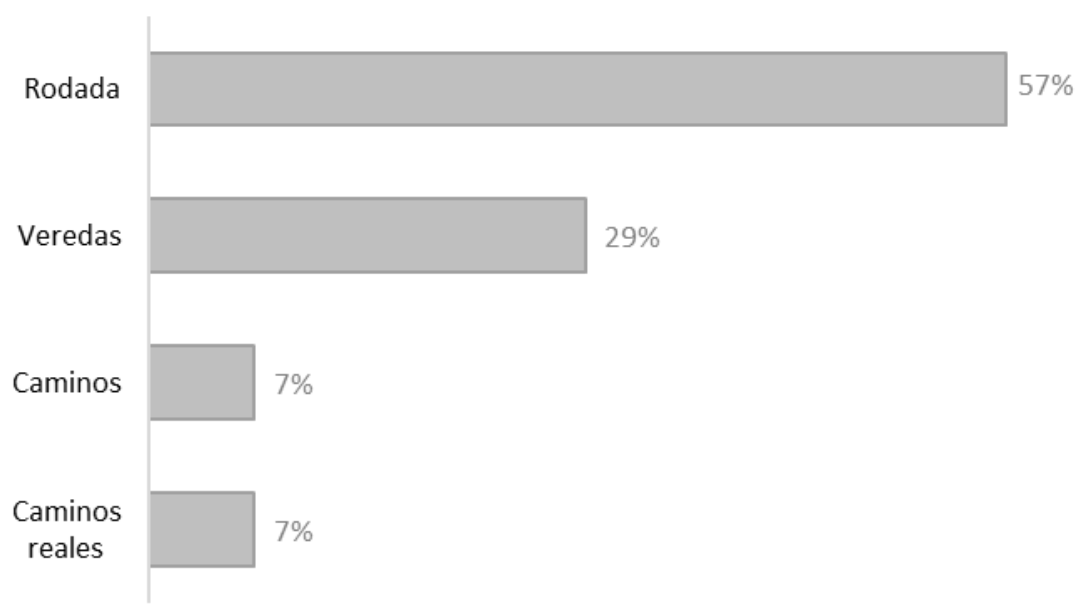

Figura 3. Accesibilidad a zonas de asentamientos de apiarios en la región de estudio. Fuente: diseño a partir de la investigación de campo realizada de agosto 2015 a noviembre 2016.

Apicultores de la región de estudio reportan trabajar un sistema de producción de una abeja reina por colmena con la Apis mellifera y con subespecies originarias de Europa, Asia y las modificadas genéticamente, de los cuales $73 \%$ de los productores realizan la 'explotación tradicional' enfocada a la producción de miel, cera y núcleos y $27 \%$ la 'explotación integral' que busca obtener ingresos adicionales a partir de obtener otros productos como polen, jalea real, propóleos, apitoxina, mieles monoflorales, orgánica, además de servicios de polinización. De acuerdo a su infraestructura y nivel de producción, ocasionalmente se apoyan de personas con actividad meramente agrícola, quienes asisten al apicultor por un ingreso extraordinario al de sus actividades del campo.

El cambio de uso del suelo, la falta de capacitación y organización de los apicultores, así como la presencia esporádica de enfermedades como la varroasis, aunado al intermediarismo y competencia en el mercado nacional, ha ocasionado inestabilidad del sector apícola de la región, lo que de alguna forma deja de ser atractiva esta actividad de manera formal (Contreras et al., 2016). Cuando menos $90 \%$ de los apicultores son campesinos de bajos ingresos económicos los que complementan con la apicultura y por lo general, aun y cuando trabajan entre 15 y 80 colmenas, ellos carecen de algún tipo de administración de sus actividades. Este perfil de apicultor no realiza registros de producción, ni de gastos de producción e ingresos.

Entre $4 \%$ y $10 \%$ son los apicultores que han invertido en la diferenciación del producto, estrategias y canales de comercialización de la miel. La edad promedio de los apicultores directamente involucrados en la actividad apícola es de 52 años. La Figura 4 muestra que $22 \%$ de los apicultores de la región de estudio tiene entre 26 y 35 años, 32\% tienen edades entre los 36 y 45 años, 19\% representan a apicultores con edades entre los 46 y 55 años, 23\% de ellos tienen edades entre los 56 y 65 años y sólo $4 \%$ representan una población entre los 66 y 75 años. 


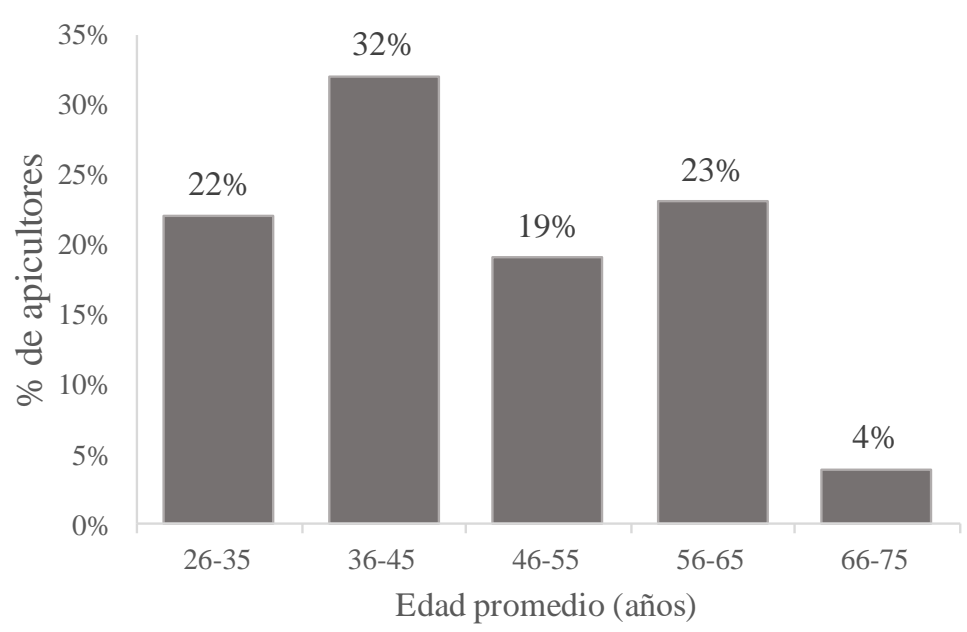

Figura 4. Promedio de edad de los apicultores de la región. Fuente: investigación de campo realizada de agosto 2015 a noviembre 2016.

El promedio de experiencia de los apicultores es de 22 años; sin embargo, la experiencia dentro de la actividad no es necesariamente un indicador del grado de especialización y profesionalización de la cadena apícola. Puede observarse en esta proporción de edades, que el rango de edad de 36 a 45 años del apicultor es la de mayor frecuencia, lo cual de alguna manera demuestra que el interés en la práctica apícola permanece vigente en este sector agrícola.

Según Reyes et al. (2014); Magaña et al. (2016), el rendimiento de producción de miel por colmena está sujeta a una serie de factores como pueden ser, la fortaleza de los núcleos de la colmena; una buena fuente de alimento; la aplicación de buenas prácticas apícolas, entre otros factores como la temporalidad la cual se manifiesta en el volumen del flujo del néctar y polen, dado éste por la disponibilidad de la flora melífera y nectarífera de la zona en que se asiente el apiario, en tal sentido, la falta de disposición de flora melífera y polinífera, propicia la trashumancia, practicada propiamente en diferentes zonas de la región de estudio.

Para identificar nuevos emplazamientos, el apicultor recorre distancias considerables en sus vehículos para que de manera visual ubique terrenos según su experiencia y posteriormente reunirse con el dueño y llegar a un acuerdo por la colocación de sus colmenas. En tal sentido, 100\% de los apicultores entrevistados coinciden en la importancia de disponer de herramientas que les agilice ubicar zonas óptimas para instalar sus apiarios.

Cuando la actividad apícola es la principal actividad económica de ingresos, se observa una relación directa con el número total de colmenas por apicultor con un promedio de 172 colmenas, desde 101 hasta 300 colmenas. En contraste cuando no es su principal actividad económica, los apicultores trabajan con 15 a 30 colmenas, por lo que buscan diversificar sus actividades de ingreso económico, mientras que los que trabajan con 31 y hasta 80 colmenas, el producto de la cosecha de miel representa entre $60 \%$ al $75 \%$ del ingreso del sustento familiar.

La apicultura presenta la fuente principal de ingresos económicos para los productores que tienen más de 500 colmenas ( $8 \%$ en la región de estudio), lo que implica un mayor movimiento de recursos a mayor número de colmenas. Por otro lado, los productores que cuentan con un número 
reducido de colmenas, esta actividad les genera bajos ingresos y los costos de producción que superan los ingresos por venta de los productos de la colmena, sin embargo, mantienen la actividad como tradición familiar para el consumo particular y familiar. Hemos encontrado que, de manera general, los apicultores coinciden en que el nivel de tecnología implicada en su práctica apícola está relacionado con el nivel económico de cada apicultor, toda vez que ésta, no es su actividad agrícola de ingreso familiar, sino más bien complementaria.

Algunos investigadores como Contreras et al. (2013); Martínez et al. (2017); Vélez et al. (2016), reportan que los apicultores en México se catalogan en tres grupos: tecnificado, semi tecnificado y tradicional. Dentro del primero están los apicultores con más de 100 colmenas (2\% de los productores nacionales) que incorporan adelantos tecnológicos de vanguardia e incluso generan su propia tecnología acorde a las características de la región. Manejan una apicultura diversificada y practican la trashumancia en busca de floraciones y mejores espacios para colocar sus apiarios. Dentro de esta categoría se observó que $9 \%$ de los productores de la región de estudio son tecnificados.

La segunda categoría la conforman los apicultores con diferentes grados de tecnificación y generalmente poseen entre 60 y 100 colmenas ( $7 \%$ de los productores nacionales) que para el caso de la zona de estudio representa $36 \%$ de los productores. Finalmente, el tercer grupo practica la apicultura como una actividad complementaria a otras labores o bien como pasatiempo. Éstos no incorporan tecnología y la mayor parte de ellos emplea técnicas rudimentarias (91\% de los productores nacionales), esta categoría está representada por $55 \%$ en la región de estudio.

La Figura 5 muestra la tendencia comparativa porcentual a nivel nacional y regional de la categorización de apicultores acorde a su sistema de producción. En lo que refiere a los rendimientos de producción de miel por colmena, aun y cuando se reportan $40 \mathrm{~kg}_{\text {colmena }}{ }^{-1}$ por apicultores de la región de estudio, la AAM reporta cosechas de miel en promedio de $24 \mathrm{~kg}$ colmena $^{-1}$, con volúmenes desde sólo $15 \mathrm{~kg}_{\text {colmena }}{ }^{-1}$ hasta los $27 \mathrm{~kg}_{\text {colmena }}{ }^{-1}$.

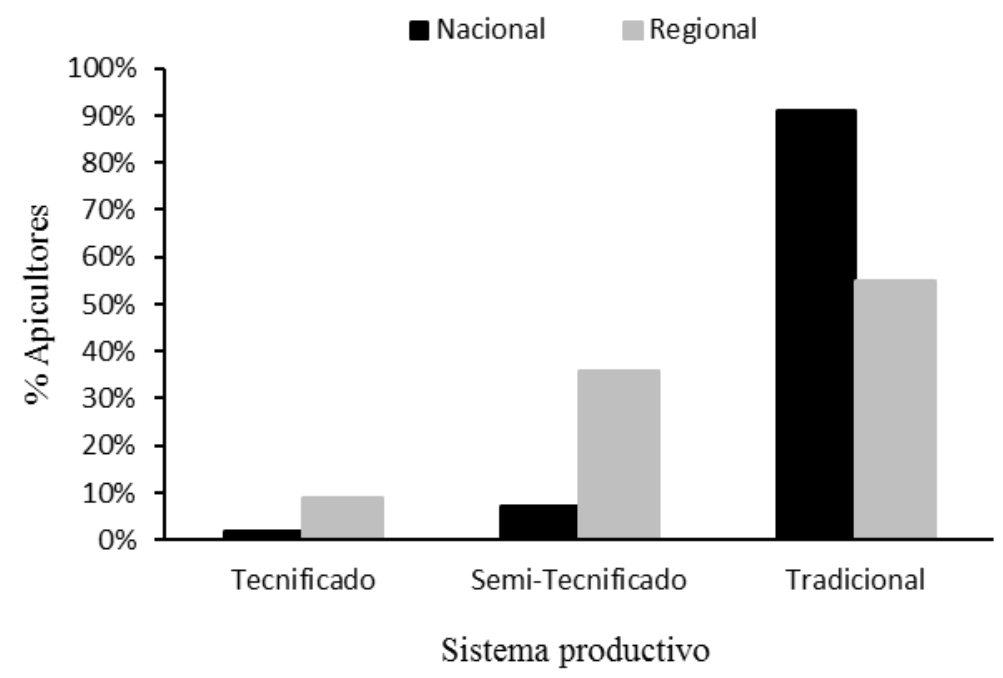

Figura 5. Comparación porcentual de la categorización del sistema productivo apícola. Fuente: SAGARPA (2017) e investigación de campo. 
La apicultura trashumante en la región incluye cuando menos 80 apicultores entre asociados e independientes. Estos apicultores son predominantes en la región intermunicipal de Misantla y constituyen un patrón de producción campesina, en donde la herencia familiar ha sido el motor que ha mantenido esta actividad agrícola. La ruta que realizan para la trashumancia en la región de estudio es en respuesta a obstáculos físicos territoriales y a la adaptación obligada con las condiciones climáticas y de floración, socioculturales e institucionales.

Entre $75 \%$ y $90 \%$ de los apicultores que practican la movilización de colmenas cuentan con rutas trashumantes ya definidas y zonas determinadas para esta práctica. De esta proporción, solamente $23 \%$ de éstos moviliza sus colmenas a otras entidades con el fin de cosechar mieles de diferente característica. El Cuadro 1 muestra la proporción de apicultores que practican trashumancia local, regional y estatal. Estos apicultores trashumantes consideran que existen zonas con mejores condiciones de producción dentro de la región de estudio que no han sido explotadas; sin embargo, no ingresan a ellas puesto que no cuentan con información de accesibilidad y permisibilidad a dichas zonas, dejando la oportunidad de explorar zonas potenciales para la apicultura.

Puede observarse en el Cuadro 1 que la mayor trashumancia, correspondiente a 43\%, se realiza en la región de estudio, la cual corresponde a una movilidad de entre 1500 y 2000 colmenas, mientras que $34 \%$ de los apicultores trashuman en el interior de su propio municipio, lo cual representa una movilidad de 1000 a 1200 colmenas. La trashumancia a otras regiones se realiza específicamente al Altiplano de Puebla y zonas de manglares del sur del estado de Veracruz, lo que representa la movilidad de 1800 a 2500 colmenas. En cualquiera de los casos, la trashumancia aprovecha el calendario de floración de dichas regiones y el número de colmenas, se determina por la experiencia de cada apicultor o bien por el resultado de la producción de miel del periodo pasado.

Cuadro 1. Trashumancia local, regional y estatal de apicultores de la región intermunicipal de Misantla.

\begin{tabular}{lccc}
\hline $\begin{array}{l}\text { Origen de los apicultores de } \\
\text { la región de estudio }\end{array}$ & $\begin{array}{c}\text { Trashumancia a } \\
\text { otras regiones }(\%)\end{array}$ & $\begin{array}{c}\text { Trashumancia regional } \\
(\%)\end{array}$ & $\begin{array}{c}\text { Trashumancia local } \\
(\%)\end{array}$ \\
\hline Misantla & 11 & 19 & 9 \\
Juchique de Ferrer & 4 & 12 & 10 \\
Yecuatla & 5 & 10 & 8 \\
Colipa & 3 & 2 & 6 \\
Tenochtitlan & 0 & 0 & 34 \\
Total de apicultores de la & 23 & 43 & \\
región de estudio & & & \\
\hline
\end{tabular}

Fuente: investigación de campo realizada de agosto 2015 a noviembre 2016.

Según los datos de la AAM, los apicultores trashumantes utilizan camionetas de 1.5 a 5 t para movilizar sus colmenas a las zonas de asentamientos. Estos apicultores mueven desde 45 hasta 90 colmenas por viaje en camionetas propias. Para la movilización de las colmenas, cuando menos 90\% de estos contratan de 1 a 3 empleados temporales para la instalación de apiarios. Por otra parte, la AAM reporta que 'la explotación apícola en la región intermunicipal de Misantla se realiza de forma sedentaria, semi-extensiva y extensiva'. 
La forma Sedentaria se caracteriza por apicultores para consumo personal-familiar con apiarios fijos con no más de 19 colmenas. La forma semi-extensiva consiste en mover sus colmenas -menor a 80 colmenas- entre lugares ya conocidos, sin complicarse con problemas logísticos en su movilización. Finalmente, en la forma Extensiva -con más de 100 colmenas-, además de ubicar sus apiarios en lugares de su región, trashuman a lugares fuera del municipio o del estado buscando siempre puntos de asentamiento para disponer de nuevas fuentes de proveeduría para sus apiarios.

\section{Discusión}

Este artículo presenta hallazgos de la producción apícola y actividad trashumante de los municipios de Misantla, Yecuatla, Colipa, Juchique de Ferrer y Tenochtitlan. Hemos encontrado, al igual que Saul da Rosa et al. (2014), que la producción de miel en esta región intermunicipal es una actividad agrícola que se realiza de manera informal y secundaria como un complemento de ingresos económica de manera familiar. En este contexto, los apicultores en esta investigación coinciden en que la utilidad económica de esta práctica agrícola está determinada, por una parte, por el nivel tecnológico implicado en su práctica apícola en el proceso de producción miel; así también, por los mecanismos de comercialización de los productos obtenidos en dicha producción de miel, como se menciona en (Simone et al., 2016).

Estos aspectos son coincidentes con Saeed-Khan et al. (2009), quienes reportan que los costos de producción y los ingresos que se perciban por la venta de miel denotan el beneficio lucrativo de esta actividad, lo cual puede ser revertido por la adecuada aplicación tecnológica en las prácticas apícolas. Carreck et al. (1997) reportan que la relación de la agricultura y la apicultura son altamente interdependientes, y que se necesita de la interacción de los agricultores y apicultores en un beneficio mutuo, para la producción de alimentos y producción de miel.

Este beneficio motiva mover las colmenas a sitios adecuados de asentamiento para el pecoreo de las abejas y aprovechar la floración disponible. Si bien el apicultor dispone de un calendario de floración de los cultivos de la región, lamentablemente esta herramienta de consulta no ha sido actualizada desde hace más de trece años, toda vez que las estaciones de floración de hoy en día han cambiado considerablemente en su calendario. Dado que no existe una técnica de selección precisa para determinar las zonas adecuadas para ubicar el apiario, el apicultor utiliza su experiencia sin evaluar si esa zona será la adecuada para la producción de la miel en el próximo periodo de producción.

El apicultor remedia este problema consultando con otros productores la estacionalidad de floración en las zonas ya utilizadas anteriormente para su explotación o bien con los campesinos de la zona de interés con el propósito de reducir los costos implicados por el acceso, los permisos de los propietarios en los puntos de instalación y así evitar la cercanía de los apiarios a zonas de población urbana. Esta experiencia la traduce para realizar trashumancias cortas y aprovechar la floración local y regional. Este esquema de manejo de apiarios, para aprovechar la flora apícola disponible en cada región, es recomendado por (Fagúndez et al., 2016).

Como estrategia de trashumancia, los apicultores disponen de un mapa que ha venido actualizando con información de sus mismos miembros para identificar los asentamientos de los apiarios por apicultor para instalar sus colmenas y no interferir con las zonas de pecoreo que ya tenían asignados 
los apiarios instalados. Sin embargo, estos mapas no muestran las zonas con potencial de proveeduría de floración, por lo que la decisión de emplazamiento la toman platicando directamente con los dueños de las parcelas, analizando de manera visual si la zona es apta o no, según su criterio y experiencia.

En este sentido, el desconocimiento mismo de la densidad de flora entre las estaciones del año y el desconocimiento de la fenología de floración presenta a la actividad de trashumancia como una situación de 'buena suerte', lo que hace de ella una actividad económicamente costosa al obtenerse bajos rendimientos de producción de miel por colmena y apiario, por abajo del promedio regional de $24 \mathrm{~kg}$ de miel por colmena.

\section{Conclusiones}

La apicultura en la región sierra norte-centro de Veracruz enfrenta un panorama de oportunidades y retos, originado principalmente de las demandas del mercado, así como de la necesidad de fortalecer la economía y la organización de los productores apícolas como principales ejes de acción que impulsen la milenaria actividad de la apicultura.

Las estrategias de supervivencia y adaptación que implementan los apicultores de la región de estudio son tan diversas como los cambios inciertos del clima y desfasamiento floral, propiciados en mayor o menor grado por la invasión de zonas de cultivo intensivos en zonas que no pudieron imaginarse en el pasado para su explotación comercial, sumando a ello el crecimiento no regularizado de la mancha urbana. Todos ellos tienen en común una brecha relacionada con el uso de tecnologías, como pueden ser, los sistemas de geoposicionamiento disponible en sus celulares, el cual no lo utilizan con dicho propósito en su práctica apícola.

Sin embargo, las estrategias que se han identificado y reportado por varios apicultores con más de 150 colmenas son: diversificación, trashumancia, intensificación y fortalecimiento de núcleos.

Los retos para la miel veracruzana en el contexto actual son asegurar la calidad y trazabilidad de la miel, obtener denominaciones de origen para mejorar el precio de la miel específica de un tipo de flora y ofrecer un producto que apoye el manejo sustentable de los bosques nativos y cultivados. En este sentido, sumado a los rendimientos de producción de miel por colmena, toma relevancia el identificar las zonas de floración que provean el suministro de néctar y polen a las abejas para la elaboración de mieles.

Así como, se requiere una mejor estrategia para realizar las actividades de trashumancia apícola como puede ser el uso de los sistemas de información geográfica (SIG) con dirección a una apicultura de precisión.

Identificar zonas espaciales para el asentamiento de apiarios, así como un programa de trashumancia entre dichos puntos potenciales de asentamientos de apiario, ha motivado la presente investigación para que, apoyado de la programación matemática, técnicas de ruteo y GIS, se presente un mapa de interés apícola para la ubicación de apiarios en la región centro-norte del estado de Veracruz. 


\section{Agradecimientos}

Por las facilidades e información proporcionada, los autores extienden su agradecimiento a los apicultores citados en esta investigación, a la Mtra. Laura Medina de SEMARNAT por su gestión ante organismos y de manera especial, el primer autor agradece al CONACYT por la beca de estudios \#687862 otorgada de agosto 2015 a septiembre 2017, para realizar los estudios de Maestría en Ingeniería Industrial en el ITS de Misantla.

\section{Literatura citada}

Abou, S. H. F.; Al, G. A. A. and Mohamed, A. A. A. 2013. Suitability map for keeping honeybees under harsh environmental conditions using geographical information system. World Appl. Sci. J. 22(8):1099-1105.

Beekman, M. and Ratnieks, F. L. 2001. Long-range foraging by the honeybee, Apis Mellifera L. Functional Ecology. 14:490-496. https://doi.org/10.1046/j.1365-2435.2000.00443.x.

Bellarby, J.; Foereid, B.; Hastings, A. and Smith, P. 2008. Cool farming: climate impacts of agriculture and mitigation potential. In: Campaigning for Sustainable Agriculture. (Ed.). greenpeace.org. JN 102. Greenpeace International. The Netherlands. 20-25 pp.

Carreck, N. L.; Williams, I. H. and Little, D. J. 1997. The movement of honeybee colonies for crop pollination and honey production by beekeepers in Great Britain. Bee World. 78(2):67-77.

Contreras, E.; Pérez, F. A. B.; Echazarreta, C. B.; Cavazos, A. J.; Macías, M. J. y Tapia, G. J. 2013. Características y situación actual de la apicultura en las regiones Sur y Sureste de Jalisco, México. Rev. Mex. Cienc. Pec. 04(03):387-398.

Contreras, R. D.; Pérez, L. M.; Payró, D. E.; Rodríguez, G.; Castañeda, H. E. y Gómez, U. R. 2016. Comportamiento defensivo, sanitario y producción de ecotipos de Apis mellifera L. en Tabasco, México. Rev. Mex. Cienc. Agríc. 7(8):1867-1877.

Erickson, E. H.; Berger, G. A.; Shannon, J. G. and Robins, J. M. 1978. Honeybee pollination increases soybean yields in the Mississippi Delta Region of Arkansas and Missouri. J. Econ. Entomol. 4(71):601-603.

Fagúndez, G. A.; Reinoso, D. P. y Aceñolaza P. G. 2016. Caracterización y fenología de especies de interés apícola en el departamento Diamante (entre Ríos, Argentina). Bol. Soc. Argent. Bot. Argentina. 51(2):243-267.

González, A. y Sánchez, J. 2008. Caracterización botánica y geográfica de mieles. Asociación Nacional de Médicos Veterinarios Especialistas en Abejas A.C. (ANMVEA, AC). In: Congreso Internacional de Actualización Apícola. Tuxtla Gutiérrez. 15(1):28-31.

INEGI. 2010. Instituto Nacional de Estadística, Geografía e Informática. http://www3.inegi.org. $\mathrm{mx} / \mathrm{sistemas} / \mathrm{mexicocifras} / \mathrm{default}$.aspx? $\mathrm{src}=487 \& \mathrm{e}=30$.

INEGI. 2015. Instituto Nacional de Estadística, Geografía e Informática. Recursos Naturales y Climatología. http://gaia.inegi.org.mx/mdm6/?v=bgf0oje5ljk1otu0lgxv bjototyuodcxnzi sejo3lgw6yzqwmxxjnda0fgm0mdd8yzqxmhxjnde3fgm0mtg=.

Magaña, M. M. y Leyva, C. M. 2011. Costos y rentabilidad del proceso de producción apícola en México. Cont. y Admon. 235:99-119. doi: 10.22201/fca.24488410e.2011.421.

Magaña, M. M.; Tavera, C. M.; Salazar, B. L. y Sanginés, G. J. R. 2016. Productividad de la apicultura en México y su impacto sobre la rentabilidad. Rev. Mex. Cienc. Agríc. 7(5):1103-1115. 
Martínez, P. L. R.; Martínez, P. J. F. y Cetzal, I. W. R. 2017. Apicultura: manejo, nutrición, sanidad y flora apícola. Universidad Autónoma de Campeche, Campeche, México. 112 p.

Reyes, C. J.; Galarza, M. J.; Muñoz, S. R. y Moreno, R. A. 2014. Diagnóstico territorial y espacial de la apicultura en los sistemas agroecológicos de la Comarca Lagunera. Rev. Mex. Cienc. Agríc. 5(2):215-228.

Roubik, D. W. 1995. Pollination of cultivated plants in the tropics. FAO Agriculture Services Bulletin; 118. Rome, Italy. 149-156 pp.

Saeed, K. A.; Damasceno, de M. V. y Sales, L. P. 2009. Desempenho da apicultura no estado do Ceará: competitividade, nivel tecnológico e fatores condicionantes. Rev. Econ. Sociol. Rural. 47(3):651-676.

SAGARPA. 2010. Situación actual y perspectiva de la apicultura en México. In: Claridades Agropecuarias. Núm. 199. Coordinación General de Ganadería. México. 31 pp.

Salvachua, J. 1989. La trashumancia en apicultura. Ministerio de Agricultura, Pesca y Alimentación (MAPA). 15. Madrid, España. 27 p.

Saul, da R. A. H.; Foguesatto, C. R.; Rogério, É.; Müller, L. E. A. P.; Kochhann, R.; Ernestina, M. y Gutiérrez, J. 2014. Caracterización de la producción y comercialización de la miel en Brasil. Rev. Bioagroc. 7(2):1-6.

Seeley, T. D. 1995. The wisdom of the hive: the social physiology of honeybee colonies. Harvard University Press. 1. London, England. 3-39 pp.

Simone, F. M.; Li, B. H.; Huang, M. H.; Strand, M. K.; Rueppell, O. and Tarpy, D. R. 2016. Migratory management and environmental conditions affect lifespan and oxidative stress in honeybees. London. Scientific Reports. 6:1-10. Doi:10.1038/srep32023

Solomon, M. 1971. Insect pollination of crops by J. B. Free London: Academic Press. Exp. Agric. 7(4):367-368.

Vélez, I. A.; Espinosa, G. J.; Amaro, G. R. y Arechavaleta, V. 2016. Tipología y caracterización de apicultores del estado de Morelos, México. Rev. Mex. Cienc. Pec. 7(4):507-524.

Visscher, P. and Seeley, T. 1982. Foraging estrategy of honeybee colonies in a temperature deciduous forest. Ecology. 63(6):1790-1801.

Zoccali, P.; Malacrinò, A.; Campolo, O.; Laudani, F.; Algeri, G.; Giunti, G.; Strano, C. P.; Beneli, G. and Palmeri, V. 2017. A novel GIS-based approach to assess beekeeping suitability of Mediterranean lands. Saudi J. Biol. Sci. 24(5):1045-1050. 